\title{
Effect of Renal Denervation on Recurrence of Atrial Fibrillation: A Meta-Analysis
}

\author{
Muhammad Shabbir Rawala ${ }^{1}$, Anum Asif ${ }^{2}$, Waqas Siddiqui ${ }^{3}$, and Aravinda Nanjundappa ${ }^{1}$ \\ ${ }^{1}$ Charleston Area Medical Center \\ ${ }^{2}$ University of Pittsburgh Medical Center \\ ${ }^{3}$ Orange Park Medical Center
}

October 26, 2020

\begin{abstract}
Renal denervation $(\mathrm{RDN})$ is a novel percutaneous procedure that reduces the sympathetic activity to the atria and the systemic blood pressure, both of which can potentially decrease atrial fibrillation (AF) recurrence. Pulmonary vein isolation (PVI) via radiofrequency or cryoablation is performed in treatment-refractory patients with atrial fibrillation. We performed a systematic review and meta-analysis to study the effects of RDN on AF recurrences with PVI vs. isolated PVI. We searched PubMed, Medline, and Google Scholar database from January 01, 2005, to January 01, 2020, for randomized control trials (RCTs) that compared the PVI for atrial fibrillation treatment with or without RDN. The primary endpoint was freedom from AF recurrence with a follow-up period of 12 months. We selected 5 RCTs comprising a total of 496 patients (249 in RDN+PVI arm and 246 in PVI only arm). RevMan Version 5.3 Copenhagen was used to calculate the relative risk (RR) of dichotomous data using a random-effects model for our review and analysis. The use of RDN in addition to PVI lead significantly more patients who were free from AF recurrence compared to PVI alone at 12 months (173 vs. 119; RR=2.70; 95\% confidence interval (CI) $=1.59-4.59$, $\mathrm{p}=0.0002, \mathrm{I} 2=36 \%$ ). The analysis showed a significant benefit of RDN addition to PVI in reducing the AF recurrence. This is an interesting finding which needs further investigation to evaluate the safety of concomitant RDN and PVI and effect on long-term survival.
\end{abstract}

\section{Hosted file}

RDN AFIB complete with tables.pdf available at https://authorea.com/users/370253/articles/ 488933-effect-of-renal-denervation-on-recurrence-of-atrial-fibrillation-a-meta-analysis 